surfaces - but at least it can be said that they perform !

The search for a homogeneous photochemical device capable of producing chemical fuel is only beginning. Europium and samarium have been used to evolve $\mathrm{H}_{2}$, but they are such rare substances that the findings are of purely academic interest. Organic materials and the use of isomerization remain to be studied. In all this, man must not attempt to imitate the results of natural evolution such as biological photosynthesis. But the study of biological and biochemical processes could well lead to the development of a practical device based on the photofixation of $\mathrm{CO}_{2}$ in a nonbiological system, perhaps 15 to 20 years from now.

With so much theory and speculation being devoted to the solar energy problem its impact on everyday life now should not be forgotten. Take black beans, for instance. J. Meyer reporting on solar energy investigations in Brazil showed that his young research group had followed the rule that science in a developing country should be closely related to general activities there. The drying of black beans is an essential part of their food economy, and it is worth remembering that $30 \%$ of the foodstuffs in the tropics perish because of the lack of correct treatment. So they developed a solar collector of $8-10 \mathrm{~m}^{2}$ in area connected by a ventilating system (so far electrically powered) to the silo full of beans. With a potential solar input of about $800 \mathrm{~W} / \mathrm{m}^{2}$ during six hours a flow of $400 \mathrm{~m}^{3} /$ hour of dry air between 16 and $30^{\circ} \mathrm{C}$ can be produced ensuring rapid drying without risk of damaging the fruit. $A$ similar system may be developed for the drying of fish.

In considering solar energy in its relations to our habitat J.K. Page dealt with conditions prevailing at higher latitudes, particularly in the UK not exactly renowned for high solar input (1975 excepted). Because the supply of solar energy and demand are controlled by meteorological factors and are grossly mismatched at high latitudes, other sources of energy have to help out. Therefore thermal storage and the prevention of heat losses are important criteria which unfortunately appear to have been ignored by many modern architects although simple peasants already had some good ideas on the heat conservation of buildings. Well informed collaboration between architects and solar technologists is thus essential. The magnitude of the task becomes clear when it is realized that the solar heat input in the UK ranges over two orders of magnitude between a good day in summer and a bad day in winter. The summer surplus should be adequate to cover winter needs, if only a good storage technique could be devised. For short-term storage the use of heavy thermal mass building materials is helpful, but even more important is the conservation of energy in buildings, at present largely lost by bad ventilation and insulation. With better insulation internal heat sources, such as even the occupants at $100 \mathrm{~W}$ per person, could make a bigger contribu- tion. Ideally windows should become valves allowing radiant energy to penetrate and preventing heat from escaping, and their orientation can have a considerable regulating effect. For the solar house of the future research should go into developing high-efficiency collectors to provide sufficient energy at high latitudes with overcast sky by using thermal siphoning with radiatively selective absorbing surfaces and the suppression of convection losses. The recovery of energy from waste water either directly or by heat pump could also be of economic benefit.

About ten years ago St. George's School at Wallasey, England, was constructed incorporating some of the above principles such as window orientation, thermal insulation and thermal storage in the building materials so that most of the heat can be obtained directly from the sun without auxiliary collectors. The school has since operated successfully on the energy from the sun, the children (and teaching staff) and electric lights, without the use of additional fossil fuel for heating. Stepping out from the crowded lecture theatres at Magurele into the bright warm sunshine with which the Conference was appropriately blessed all the week, one wondered, had the splendid modern conference building been equipped in line with the aims of solar technology, what surplus energy could have been produced by the sun and all those speakers and participants, if only the problem of storage were solved.

W.S.N.

\title{
Transport and Storage of Energy
}

Energy strategies can depend as much on distribution and manipulation as on the availability of particular primary sources. From predictive studies, taking into account these factors, C. Marchetti deduced that, at least in the USA, the fuel most likely to be affected by any big increase in nuclear power capability was gas rather than oil or coal. Extending predictions still further into the future he forecast a rapid decline in nuclear energy usage in favour of solar energy around the year 3050 !

While admitting that mankind has no real influence on the availability of primary sources of energy, our influence on the means of transporting and using energy was paramount. The ease of transporting a particular form of energy affected the scale of the plant for producing it and, therefore, the economics which could be achieved by the use of larger plants, and fewer plants, an example being the growth in size of electric power plants and their relative decline in numbers as the ability to transmit electricity over large distances has improved. While geographical factors have played a major part, it can be seen that the ability to transport oil has grown apace with its use. Natural gas if available in adequate quantities could become a world commodity dispensed from a few very large fields with ease and economy by pipeline, and stored locally in the many geologically favourable underground sites.

The ease of transporting a particular form of energy really determines whether the strategy is many power sources situated close to the points of consumption or a few large ones supplying very large areas whilst the technology of energy transport is bounded by fundamental physical and economic constraints. The practical distance limit of transmission for each form of energy determines the optimum size of the power plant, e.g. hot water: $2 \mathrm{~km}$ 0.2GW, electricity: $100 \mathrm{~km}$ 1GW; hydrogen: $1000 \mathrm{~km}, 100 \mathrm{GW}$; ADAM/EVA (nuclear generated methane): $2-3 \times 10^{3} \mathrm{~km}, 10^{3} \mathrm{GW}$; Oil: $10^{4} \mathrm{~km}, 2 \times 10^{3} \mathrm{GW}$ (typical oil field).

\section{Electricity Transmission}

Developments arrived at improving the efficiency of electricity transmission (A. Rocher) are pointing towards UHV overhead transmission, improved conventional, cryogenic and superconducting cables underground. A key issue in this is the cost, which depends on the cable loading requirements.

For electrical power transmission in excess of $500 \mathrm{MW}$ per cable, super- 
conducting cables would be needed and both $D C$ and $A C$ forms were being developed. According to $P$. Penczynski there were no problems with thermal insulation and cryogenic techniques, but there was need for development of the superconducting alloys and the electrical insulation. $\mathrm{He}$ discussed the relative merits of $\mathrm{NbTi}$ and $\mathrm{Nb}_{3} \mathrm{Sn}$ alloys in the light of the overall requirements of as high a critical current density and as high a transition temperature as possible. The tin alloy has the advantage of ease of manufacture and better performance under fault conditions. Multilayer techniques for stabilising the conductors against transient temperature rises due to current overloads are employed.

Differential thermal expansion of the cable components presented constructional problems since plastic deformation of the conductors must be avoided. For rigid cables this could be overcome with $\mathrm{NbCu}$ - invar tubes as an outer casing with vacuum or liquid helium as the dielectric material, though both methods placed rather limiting restrictions on the electrical stress which could be imposed. Improved electrical breakdown resistance could probably be achieved by pressurisation of liquid helium. For flexible cables, electrical insulation was achieved with polyethylene and PTFE tapes, other polymers being too lossy. In these polymer tape insulations partial discharges occur at stresses much lower than that of electrical breakdown, and it was necessary to suppress these by helium pressurisation. Longer term idevelopments might see the use of liquid hydrogen as the coolant since helium was scarce, and the NiGe alloy which has a high transition temperature $(22 \mathrm{~K})$ might become generally available as a practical superconducting material.

\section{Storage}

For the storage of energy in large electrical systems pumped storage is the most advanced, but compressed air (with or without heat storage) and hot water storage are likely to be attractive alternatives. Tankers or pipelines can transport fluids at elevated temperatures $\left(<200^{\circ} \mathrm{C}\right)$ and such systems might be attractive at distances $>10 \mathrm{~km}$.

For all these approaches to energy storage other than pumped storage, Rocher foresaw the need for a good deal of development work but he was skeptical about the large scale application of lead-acid, sodium-sulphur or other types of battery even in the long term.

\section{Eleciric Batteries}

The requirements on electric batteries vary in scale from bulk energy storage as part of a generating system covering peak load situations to limited range urban transport. W.T. Eeles characterised the various possible systems in terms of their storage efficiency as functions of weight and volume, concluding that the best options lay in the use of battery components of the lighter highly reactive elements.

One particularly promising development is the Na-S battery, which depends crucially for its operation on the unique electrical and good mechanical properties of the $\beta$-alumina (nominally $\mathrm{Na}_{2} \mathrm{O} .11 \mathrm{Al}_{2} \mathrm{O}_{3}$ ) solid electrolyte used. So far, successful running of a small vehicle has been idemonstrated, for ranges of $150 \mathrm{~km}$.

On other possible developments, Eeles expressed the view that the $\mathrm{Zn}-\mathrm{Cl}_{2}$ cell with chlorine stored as solid $\mathrm{Cl}_{2} 6 \mathrm{H}_{2} \mathrm{O}$ holds promise.

\section{Fuel Cells}

A. Winsel discussed the materials problems of low temperature $\left(\sim 80^{\circ} \mathrm{C}\right)$ fuel cells. These centred around the electrode materials and particularly the catalysts needed to induce the reactants $\mathrm{H}_{2}$ and $\mathrm{O}_{2}$ to enter aqueous solutions as ionised species. Ideally the fuels for a fuel cell should be liquids as storage of gaseous fuels involved bulky and heavy pressure vessels. Therefore there is much interest in developing liquid fuels from which hydrogen can be released by either high temperature processes or by catalytic action. Here platinum dispersed in a porous nickel electrode could be used to dissociate the lighter paraffins while alcohols could be decomposed to give $\mathrm{H}_{2}$ and $\mathrm{CO}_{2}$, with WC catalysts. The $\mathrm{CO}_{2}$ must be removed as it would contaminate the alkaline electrolyte of the fuel cell. There were various ways also of using ammonia either directly or after conversion to hydrazine. The design of electrode pore structures which will ensure adequate catalyst availability at the three phase interface, where the dissociative and electron transfer processes occurred are highly complex. Practical fuel cells which represent an advance on the Bacon cell in terms of compactness and suitability for vehicles are being developed. Should hydrogen become readily available as a secondary fuel Winsel felt it would be logical to transport it as liquid ammonia since it is much easier to store than liquid hydrogen.
Rapid publication of research in physics

\section{A new physics letters journal}

Communications on Physics is a new international physics letters journal commencing publication in January 1976. It will provide rapid publication of important new work in all areas of pure physics apart from nuclear and high energy physics.

\section{Established readership}

The journal will be sent free of charge during the whole of 1976 to individual and library subscribers to Philosophical Magazine, Advances in Physics and Molecular Physics and to any library that requests it. An immediate circulation to the physics community throughout the world is thereby assured.

\section{Acceptance for publication}

Papers may be in English, French or German and should reach the Editors from 1st October 1975. Maximum length is 2500 words. Copies of Instructions to Authors may be obtained from the Editors or from the publishers.

\section{Editors and}

\section{Editorial Board}

David Caplin and David Sherrington Department of Physics,

Blackett Laboratory, Imperial College, London SW7 2BZ.

The Editorial Advisory Board is composed of distinguished physicists from all parts of Europe.

\section{Free subscription} for 1976

Librarians and others who wish to receive Communications on Physics from the first issue should write to

Miss Ann Denovan,

Taylor \& Francis Ltd,

10-14 Macklin Street,

London WC2B 5NF.

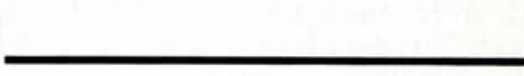 \\ Communications

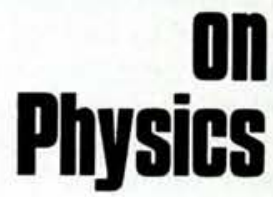

the NEW physics letters

journal from

\section{TAYLOR \& FRANCIS}

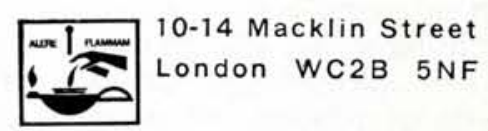


The session closed with an impromptu contribution by B.M. Vul (USSR), on recent developments in large superconducting magnets which provide a means of storage suitable for large pulses of electrical energy.

In a panel discussion on solid electrolytes the use of solids with abnormal high ionic mobility was considered. Their application is largely in facilitating electrochemical reactions between elements which are incompatible with aqueous electrolytes, a prime example being the use of $\beta$-alumina in the sodium-sulphur battery. Other uses have been proposed, such as refinement of an ionic species by electricity generation by stimulating ionic diffusion in the solid by means of a large temperature difference between two reservoirs of the appropriate liquid metal or vapour.

The properties of the one solid electrolyte being experimented with on a large scale were discussed, notably how its ionic conductivity $\sim 0.3 \mathrm{mho}^{-1}$ at $300^{\circ} \mathrm{C}$ was related to the existence of metastable sodium ion sites in the $\beta$-alumina lattice, and how the occupancy of such metastable sites is influenced by the occurrence of stacking faults in the lattice, together with the stabilisation of above stoichiometric concentrations by replacing some of the aluminium with a di-valent or mono-valent metal.

\section{Thermal Storage}

Of the possibilities for thermal storage in hot liquids including water, hydrates or chlorides, hot water storage seems to hold out most promise, but the capital cost of the most obvious route using pressurised vessels is still high. Possible cheaper ways include storage in the water of natural water/rock conglomerates, in lakes, in wet soil and in naturally occurring caverns filled with water. D. J. Schroeder described also storage by latent heat associated with solidification of salt solutions at near ambient temperature and highlighted $\mathrm{KF} .4 \mathrm{H}_{2} \mathrm{O}$ as a material with almost ideal properties for use in domestic solar energy heating and air conditioning systems. His high tempera- ture molten salt storage heater for indoor domestic installations has a super-insulation jacket of controllable thermal transmittance.

Most of these methods will demand considerable development effort before entering the field of practical economic operation.

\section{Coal Conversion}

A wide range of possibilities exist for conversion of coal to liquid and gaseous secondary fuel products with important advantages to the ultimate user. Whilst discussing the UK research programme on these topics, $F$. Williams emphasised the range of international developments in, for example, the Lurgi and Cogas processes. Among a number of examples for the longer term, a process for solution of coal in anthracene oil with subsequent regeneration and production of a range of organic products was interesting but again much research is going to be needed to bring these ideas into practice.

W.T.E. B.E.

\section{Nuclear Energy}

All our hopes are in nuclear energy. This statement made first by $P$. L. Kapitza in his opening talk was to be echoed on a number of occasions. Nobody is completely comfortable and would welcome another way out but there are no real alternatives in the foreseeable future to supply the massive increase in demand and particularly in electricity production that is needed. In A.M. Weinberg's words, it would be wrong to abandon nuclear energy by evoking other talismans. In exchange for an inexhaustible and clean source of energy, society promised to pay meticulous attention to its use. This last phrase had been used previously by Weinberg when speaking of the Faustian deal on nuclear energy but he was careful to explain on this occasion that this did not imply a devilish exchange, Faust was, after all, redeemed.

The problems are formidable it is admitted and it would be comfortable if mastering the fusion reaction was something that we could expect in the near future but it was difficult to anticipate a working fusion reactor before the end of the century and we were only too well aware how long after this it would be, before there might be reliable, economic and safe production units on the networks of the world.

\section{Development of the LWRs}

It was a reflection on the maturity of nuclear energy as Weinberg's talk was entitled, that he was able, whilst speaking essentially of the evolution of reactor systems in the United States, to be speaking effectively for everybody. Gone are the days where discussions on nuclear power were dominated by the strident claims of the protagonists of competitive systems and the market infighting of competing manufacturers. But Weinberg emphasized this should not mean that no reappraisal was necessary; it was highly desirable that forward policies should be studied intensively and alternative strategies prepared.

It was an accident of history that had resulted in the almost universal adoption of light water reactor systems for central power production (more than 200 are currently on order) as these haid initially been developed for submarine propulsion where compactness was the crucial criterion. They proved however, to be relatively cheap and reliable and when the cost of a unit of separated work fell by a a factor of ten between 1945 and 1960 to a figure of around $\$ 12 / \mathrm{g}$ ( the momentum they had gathered led them inexorably onwards. But whereas some years ago the capital cost of heavy water in the $\mathrm{D}_{2} \mathrm{O}$ systems imposed a serious financial disadvantage, it was no longer clear that the same conditions held good today.

\section{Breeders}

At a very early stage, the necessity for a breeder reactor was appreciated as uranium without breeding is not a very interesting source of energy in the long term. In the Fermi mind, breeding was synonymous with the fast breeder but Wigner had promoted the concept of the thermal breeder until the poisoning effect of fission products dominated by the $135 \mathrm{Xe}$ cross-section of 2,6 million barns indicated that it could only work in a homogeneous system. As a result, the FBR emerged as the only contender but it is a very different animal from the one that was first envisaged; cores are bigger, fuel elements taken to high burnups and in consequence chemical separation is done in specialized plants far away. But the FBR is not necessarily the only solution and unless it is clear that a short doubling time is vital, a light water or even heavy water breeder merits more detailed study. Liquid systems have also a lot to recommend them and Weinberg diffidently recalled the molten salt experiment which had really gone very well. 